\title{
Ergodicity for piecewise smooth cocycles over toral rotations
}

\author{
by
}

\author{
A. I wanik (Wrocław)
}

\begin{abstract}
Let $\alpha$ be an ergodic rotation of the $d$-torus $\mathbb{T}^{d}=\mathbb{R}^{d} / \mathbb{Z}^{d}$. For any piecewise smooth function $f: \mathbb{T}^{d} \rightarrow \mathbb{R}$ with sufficiently regular pieces the unitary operator $V h(x)=\exp (2 \pi i f(x)) h(x+\alpha)$ acting on $L^{2}\left(\mathbb{T}^{d}\right)$ is shown to have a continuous nonDirichlet spectrum if the gradient of $f$ has nonzero integral. In particular, the resulting skew product $S_{f}: \mathbb{T}^{d+1} \rightarrow \mathbb{T}^{d+1}$ must be ergodic. If in addition $\alpha$ is sufficiently well approximated by rational vectors and $f$ is represented by a linear function with noninteger coefficients then the spectrum of $V$ is singular. In the case $d=1$ our technique allows us to extend Pask's result on ergodicity of cylinder flows on $\mathbb{T} \times \mathbb{R}$ to arbitrary piecewise absolutely continuous real-valued cocycles $f$ satisfying $\int f=0$ and $\int f^{\prime} \neq 0$.
\end{abstract}

Introduction. Let $f$ be a piecewise absolutely continuous function on the unit interval such that $\int f^{\prime} \neq 0$ and let $\alpha \in \mathbb{R} \backslash \mathbb{Q}$. In [7] it is shown that the skew product transformation of $\mathbb{T}^{2}$ defined by

$$
S_{f}(x, y)=(x+\alpha, y+f(x)),
$$

where the addition is modulo 1 , has a continuous spectrum on the orthocomplement in $L^{2}\left(\mathbb{T}^{2}\right)$ of the functions depending only on the first variable. Moreover, if $f$ has a single discontinuity with jump $c$ then for every $k \in \mathbb{Z}$ such that $k c$ is not an integer the spectrum is singular on the invariant subspace $H_{k}=\left\{h(x) \exp (2 \pi i k y): h \in L^{2}(\mathbb{T})\right\}$ (see Theorem 2 of [7]; special cases have been proved earlier in [2] and [9]) .

By investigating the invariant subspaces $H_{k}$ the spectral analysis of the skew product reduces to that of the unitary operator $V=V_{\alpha, f}$ on $L^{2}(\mathbb{T})$, where

$$
(V h)(x)=e^{2 \pi i f(x)} h(x+\alpha)
$$

(see Anzai [1]), and in fact to the spectral measure $\sigma_{\alpha, f}=\sigma$ of the function $h=1$ :

1991 Mathematics Subject Classification: Primary 28D05.

Supported in part by KBN grant 2P 03A 07608. 
where we set

$$
\widehat{\sigma}(n)=\left(V^{n} 1,1\right)=\int e^{2 \pi i f^{(n)}},
$$

$$
f^{(n)}(x)=f(x)+f(x+\alpha)+\ldots+f(x+(n-1) \alpha)
$$

for $n \geq 1$.

In the present note we consider "piecewise smooth" real-valued functions $f$ on the $d$-torus $\mathbb{T}^{d}$ and prove that, as in dimension 1 , for any ergodic rotation $\alpha$ of $\mathbb{T}^{d}$ the measure $\sigma$ must be continuous if the integral of the gradient of $f$ does not vanish (Corollary 2). This clearly gives spectral continuity of $S_{f}$ on each invariant subspace $H_{k}, k \neq 0$ (defined as for $d=1$ ), which in turn implies ergodicity of the skew-product transformation $S_{f}$ on $\mathbb{T}^{d+1}$. Our proof gives a little more also in the one-dimensional case as we find that the spectral measure $\sigma$ can never be a Dirichlet measure.

For linear functions with all noninteger coefficients we only observe that for "most" rotations the spectrum is singular (Theorem 2), leaving the general case unresolved.

Finally, in the one-dimensional case $d=1$ we indicate how to apply our technique to modify Pask's argument in [10]. As a result we obtain an extension of his ergodicity result to the cylinder flows on $\mathbb{T} \times \mathbb{R}$ determined by arbitrary real-valued piecewise absolutely continuous cocycles $f$ satisfying $\int f=0$ and $\int f^{\prime} \neq 0$ (Theorem 3 ).

Throughout the paper we use notation and terminology of [7].

1. Spectral continuity. Let $(X, \mu),(Y, \nu)$ be probability spaces and $R, T$ be invertible measure preserving transformations acting on $X, Y$, respectively. For $n \geq 1$ we write

$$
g^{(n)}(x, y)=\sum_{j=0}^{n-1} g\left(R^{j} x, T^{j} y\right) .
$$

Lemma 1. If the direct product $R \times T$ is ergodic then for every $g \in$ $L^{1}(\mu \times \nu)$ there exists a set $B$ of measure 1 in $Y$ such that

$$
\int\left|\frac{g^{(n)}(x, y)}{n}-\int g d(\mu \times \nu)\right| d \mu(x) \rightarrow 0
$$

for all $y \in B$.

Proof. We set

$$
A_{n} g(x, y)=\frac{g^{(n)}(x, y)}{n}-\int g d(\mu \times \nu) .
$$

The individual ergodic theorem implies $A_{n} g \rightarrow 0$ a.e. on $X \times Y$ for every $g \in L^{\infty}(\mu \times \nu)$. Therefore, by Fubini's theorem, for almost every $y$ the bounded convergence $A_{n} g(x, y) \rightarrow 0$ holds for $\mu$-a.e. point $x$. Now the 
Lebesgue bounded convergence theorem implies that the lemma holds for every $g \in L^{\infty}(\mu \times \nu)$.

Since $L^{\infty}$ is dense in $L^{1}$, for every $g \in L^{1}$ and $\varepsilon>0$ there exists $g_{0} \in L^{\infty}$ such that $\int g_{0} d(\mu \times \nu)=\int g d(\mu \times \nu)$ and $\left\|g-g_{0}\right\|_{1}<\varepsilon / 2$. We let

$$
h(y)=\int\left|g(x, y)-g_{0}(x, y)\right| d \mu(x) .
$$

Clearly, $\|h\|_{L^{1}(\nu)}<\varepsilon / 2$, so by the ergodicity of $T$ we have, for a.e. $y$,

$$
\frac{1}{n} \sum_{j=0}^{n-1} h\left(T^{j} y\right)<\frac{\varepsilon}{2}
$$

if $n \geq n_{0}(y)$. Moreover, by the first part of the proof we may assume that $\int\left|A_{n} g_{0}(x, y)\right| d \mu(x)<\varepsilon / 2$ for $n \geq n_{0}(y)$. Consequently,

$$
\begin{aligned}
\int\left|A_{n} g(x, y)\right| d \mu(x) & \leq \int\left|\frac{g^{(n)}(x, y)-g_{0}^{(n)}(x, y)}{n}\right| d \mu(x)+\int\left|A_{n} g_{0}(x, y)\right| d \mu(x) \\
& <\frac{1}{n} \sum_{j=0}^{n-1} \int\left|g\left(x, T^{j} y\right)-g_{0}\left(x, T^{j} y\right)\right| d \mu(x)+\frac{\varepsilon}{2} \\
& =\frac{1}{n} \sum_{j=0}^{n-1} h\left(T^{j} y\right)+\frac{\varepsilon}{2}<\varepsilon
\end{aligned}
$$

for $n \geq n_{0}(y)$, which ends the proof of Lemma 1 .

The following theorem applies to ergodic rotations of $\mathbb{T}^{d}$ and to piecewise smooth functions $f$ on $\mathbb{T}^{d}$, but we prove it in a greater generality. As usual, we identify the unit circle $\mathbb{T}$ with the interval $[0,1)$. Lebesgue measure is denoted by $\lambda$.

Theorem 1. Let $R x=x+\alpha$ be an irrational rotation of $\mathbb{T}$ and $T$ be any invertible measure preserving transformation of a probability space $(Y, \nu)$ such that the direct product $R \times T$ is ergodic. Let $f: \mathbb{T} \times Y \rightarrow \mathbb{R}$ be a measurable function satisfying the following four conditions:

(1) for a.e. $y$ the function $f(\cdot, y)$ is piecewise absolutely continuous on $[0,1)$ with $N(y)$ pieces,

(2) $\int N(y) d \nu(y)<\infty$,

(3) $\partial f / \partial x \in L^{1}(\mathbb{T} \times Y)$,

(4) $\iint(\partial f / \partial x)(x, y) d x d \nu(y) \neq 0$.

If $Z$ is any set of real numbers such that $\inf \{|u-v|: u, v \in Z, u \neq v\}>0$ then

$$
\lim _{\delta \rightarrow 0} \limsup _{n}(\lambda \times \nu)\left\{(x, y): f^{(n)}(x, y) \in Z+(-\delta, \delta)\right\}=0 .
$$

In particular, no subsequence of $f^{(n)}$ can converge to $Z$ in measure. 
Proof. Without loss of generality we may assume that the value of the double integral in (4) is $c>0$, that the integral in (2) is strictly less than a natural number $M$, and that any two distinct points of $Z$ differ by at least $\eta>0$. Choose a large integer $L$ (to be specified later) and a positive number $\varepsilon \leq 1 / L$. We also fix a positive number $\gamma$. Since $T$ is ergodic, we have

$$
\lim \frac{1}{n} \sum_{j=0}^{n-1} N\left(T^{j} y\right)<M
$$

for a.e. $y$. Let $B$ be a set of measure 1 in $Y$ on which (1) as well as the latter condition and the assertion of Lemma 1 for

$$
g(x, y)=\frac{\partial f}{\partial x}(x, y)
$$

hold true. We fix a point $y \in B$. There exists a minimal positive integer $n(y)$ such that

$$
\sum_{j=0}^{n-1} N\left(T^{j} y\right)<M n
$$

for all $n \geq n(y)$. This implies that the function $f^{(n)}$ is piecewise absolutely continuous with at most $M n$ continuity intervals. Those among them which have length less than $1 /(L M n)$ cover at most $1 / L$ of the unit interval. By subdividing each of the remaining longer intervals into parts of length exactly $1 /(L M n)$ plus a remainder interval, we may assume that the collection of continuity pieces for $f^{(n)}$ consists of "long" intervals of length $1 /(L M n)$ and "short" ones of a smaller length. Clearly, the long intervals cover more than $1-2 / L$ of $[0,1)$. Denote by $\mathcal{P}$ the resulting partition of the unit interval and by $\mathcal{E}$ the corresponding conditional expectation.

By Lemma 1 and the choice of $y$, there exists a minimal positive integer $n_{1}(y) \geq n(y)$ such that

$$
\int\left|\frac{g^{(n)}(x, y)}{n}-c\right| d x<\varepsilon^{2}
$$

for all $n \geq n_{1}(y)$. This means

$$
\int \mathcal{E}\left|\frac{g^{(n)}(x, y)}{n}-c\right| d x<\varepsilon^{2}
$$

so $\mathcal{E}\left|g^{(n)}(\cdot, y) / n-c\right|<\varepsilon$ on a set $A$ of measure at least $1-\varepsilon$. The intervals from $\mathcal{P}$ which form the set $A$ will be called "good". Since $\varepsilon \leq 1 / L$, it is clear that good long intervals cover at least $1-3 / L$ of the interval $[0,1)$.

For every (open) long good interval $I$ and $n \geq n_{1}(y)$ we have

$$
\int_{I}\left|g^{(n)}(x, y)-n c\right| d x<\varepsilon|I| n=\varepsilon /(L M)
$$


Denoting the left endpoint of $I$ by $a$ and letting $b=f^{(n)}(a+, y)-n c a$ we obtain

$$
\begin{aligned}
\left|f^{(n)}(x, y)-n c x-b\right| & =\left|\int_{a}^{x} g^{(n)}(t, y) d t-\int_{a}^{x} n c d t\right| \\
& \leq \int_{a}^{x}\left|g^{(n)}(t, y)-n c\right| d t<\frac{\varepsilon}{L M}
\end{aligned}
$$

for all $x \in I$.

Now it is clear that the image of $I$ by $f^{(n)}$ is an interval of length at most

$$
c n|I|+\frac{2 \varepsilon}{L M}=\frac{c+2 \varepsilon}{L M},
$$

so there are no more than $(c+2 \varepsilon) /(L M \eta)+1$ points from $Z$ in it.

On the other hand, since $\left|f^{(n)}(x, y)-(n c x+b)\right|<\varepsilon /(L M)$ on $I$, for every $\delta>0$ and $u \in \mathbb{R}$ the set $\left\{x \in I: f^{(n)}(x, y) \in(u-\delta, u+\delta)\right\}$ is contained in an interval of length

$$
\frac{1}{n c}\left(\frac{2 \varepsilon}{L M}+2 \delta\right)
$$

which simply is the $x$-projection of the parallelogram defined by the lines $l(x)=n c x+b \pm \varepsilon /(L M)$ and $l(x)=y \pm \delta$.

It follows that the points $x$ from $I$ with $f^{(n)}(x, y) \in Z+(-\delta, \delta)$ form a set of measure at most

$$
\left(\frac{c+2 \varepsilon}{L M \eta}+1\right) \frac{1}{n c}\left(\frac{2 \varepsilon}{L M}+2 \delta\right) .
$$

Consequently, the ratio of the Lebesgue measure of this set to the length of $I$ is at most $L M n$ times the above expression, which is less than $(4 / c)(\varepsilon+$ $L M \delta$ ) if $L$ is chosen sufficiently large (the choice depending only on the constants $c$ and $M$, and $\eta$ ).

Since the rest of the space has measure less than $3 / L$, we deduce that for every $n \geq n_{1}(y)$ the set of points $x$ in $[0,1)$ satisfying the condition $f^{(n)}(x, y) \in Z+(-\delta, \delta)$ has Lebesgue measure at most $(4 / c)(\varepsilon+L M \delta)+3 / L$, which is less than $\gamma / 2$ if $L$ is chosen sufficiently large, and $\varepsilon$ and $\delta$ are small enough (first fix a large $L>12 / \gamma$, then fix $\varepsilon<\gamma c / 32$, finally let $\delta<\gamma c /(32 L M))$.

By choosing the minimal possible values for $n(y)$ and $n_{1}(y)$ in the definitions above we ensured that these integer-valued functions are measurable, so there is a universal number $n_{0}$ such that $n_{1}(y) \leq n_{0}$ on a set of measure greater than $1-\gamma / 2$ in $Y$. Consequently, for $n \geq n_{0}$ the product measure $\lambda \times \nu$ of the set $\left\{(x, y): f^{(n)}(x, y) \in Z+(-\delta, \delta)\right\}$ is at most $\gamma$, completing the proof of Theorem 1 . 
Under the assumptions of Theorem 1 , let $T_{f}$ denote the skew-product transformation on $\mathbb{T} \times Y \times \mathbb{T}$ defined by

$$
S_{f}(x, y, z)=(R x, T y, z+f(x, y)) .
$$

The corresponding unitary operator $U h=h \circ S_{f}$ on $L^{2}(\mathbb{T} \times Y \times \mathbb{T})$ splits into the direct sum of operators conjugate to

$$
V_{k} h(x, y)=\exp (2 \pi i k f(x, y)) h(R x, T y), \quad k \in \mathbb{Z},
$$

acting on $L^{2}(\mathbb{T} \times Y)$. Without loss of generality we restrict our analysis to $V=V_{1}$. (We note in passing that for cocycles taking values in more general compact metric abelian groups $G$, such as e.g. $G=\mathbb{T}^{d}$, the spectral analysis is done by means of characters of $G$, so actually it reduces to the case of $\mathbb{T}$-valued cocycles as above.)

Denoting by $\sigma$ the spectral measure of the constant function 1 for $V$ we have the familiar formula for the Fourier coefficients

$$
\widehat{\sigma}(n)=\left(V^{n} 1,1\right)=\iint e^{2 \pi i f^{(n)}(x, y)} d x d \nu(y) .
$$

If $\lim \sup |\widehat{\sigma}(n)|=1$ then by the strict convexity of the unit disk there exists a subsequence $n_{j}$ such that $e^{2 \pi i f^{\left(n_{j}\right)}(x, y)}$ converges in measure to a point on the unit circle. Therefore $f^{\left(n_{j}\right)}(x, y)$ has to converge in measure to the discrete set $Z=\mathbb{Z}+u$ for some $u \in \mathbb{R}$. Now the following is clear.

COROLlary 1. Under the assumptions of Theorem 1 the Fourier coefficients $\widehat{\sigma}(n), n \neq 0$, are bounded away from the unit circle.

In the special case where $S$ is an ergodic rotation of the $d$-torus $\mathbb{T}^{d}$ determined by the vector $\left(\alpha_{1}, \ldots, \alpha_{d}\right)$, we put $Y=\mathbb{T}^{d-1}$ and $T$ the rotation of $Y$ by $\left(\alpha_{2}, \ldots, \alpha_{d}\right)$. Now $S=R \times T$, where $R$ is the $\alpha_{1}$ rotation of $\mathbb{T}$. Any piecewise smooth function $f$ on $\mathbb{T}^{d}$, even with infinitely many pieces, such that $\int \nabla f \neq 0$ satisfies the assumptions of Theorem 1 (with a possible permutation of coordinates), if only the pieces are sufficiently smooth to assure the condition (2) of Theorem 1. In particular, this is the case if the regions of smoothness are determined by sufficiently regular boundaries, as e.g. for any polygonal, algebraic, or analytic grid in $\mathbb{T}^{2}$ with finitely many pieces - since we then have $N(\cdot) \in L^{\infty}(\mathbb{T})$, so (2) clearly holds.

We say that $f: \mathbb{T}^{d} \rightarrow \mathbb{R}$ is piecewise smooth with nice pieces if conditions (1)-(3) are satisfied.

Recall that for the ergodic rotation $S$ we have the "purity law" of Helson [3]: the maximal spectral measure of $V$ is either discrete, or singular, or else equivalent to Lebesgue measure. Consequently, we may recognize the spectral type of $V$ by looking at the measure $\sigma$.

Recall also that the measure $\sigma$ is called Dirichlet if it is continuous and $\lim \sup _{n}|\widehat{\sigma}(n)|=1$. On the other hand, by Kronecker's theorem, for discrete $\sigma$ we always have $\lim \left|\widehat{\sigma}\left(n_{j}\right)\right|=1$ along some subsequence $n_{j}$. Therefore we 
obtain the following consequence of Corollary 1, strengthening Proposition 2 of $[7]$.

COROLlaRY 2. Let $f$ be a piecewise smooth function with nice pieces on $\mathbb{T}^{d}$ and assume

$$
\int_{\mathbb{T}^{d}} \nabla f \neq 0
$$

Then for every ergodic rotation $\left(\alpha_{1}, \ldots, \alpha_{d}\right)$ of $\mathbb{T}^{d}$ the spectral type of the operator

$$
V h\left(x_{1}, \ldots, x_{d}\right)=e^{2 \pi i f^{(n)}\left(x_{1}, \ldots, x_{d}\right)} h\left(x_{1}+\alpha_{1}, \ldots, x_{d}+\alpha_{d}\right)
$$

is represented by a continuous non-Dirichlet measure.

2. Cocycles represented by linear functions. Now we consider an ergodic rotation $S x=x+\alpha, \alpha=\left(\alpha_{1}, \ldots, \alpha_{d}\right)$, of $\mathbb{T}^{d}$ and a cocycle of the special type $f\left(x_{1}, \ldots, x_{d}\right)=f_{1}\left(x_{1}\right)+\ldots+f_{d}\left(x_{d}\right)$. It is clear that in this case

$$
\widehat{\sigma}(n)=\widehat{\sigma}_{1}(n) \ldots \widehat{\sigma}_{d}(n),
$$

where $\sigma_{j}$ is the spectral measure of the constant 1 function for the $L^{2}(\mathbb{T})$ operator $h \rightarrow \exp \left(2 \pi i f_{j}^{(n)}(x)\right) h\left(x+\alpha_{j}\right)$. This means that $\sigma$ is the convolution of the measures $\sigma_{j}$. Therefore if at least one of them is equivalent to Lebesgue measure, so is $\sigma$. This is the case when e.g. one of the $f_{j}$ is $C^{2}$ on the open interval $(0,1)$ and satisfies $f_{j}(1-)-f_{j}(0+) \in \mathbb{Z} \backslash\{0\}$ (see e.g. [6]).

Let us now discuss the case where all the functions $f_{j}$ are piecewise absolutely continuous on $\mathbb{T}$ with a single jump which, for all the $f_{j}$ 's, takes a noninteger value. The simplest example of this situation is a linear cocycle of the form

$$
l\left(x_{1}, \ldots, x_{d}\right)=c_{1} x_{1}+\ldots+c_{d} x_{d}+b,
$$

where $c_{j} \in \mathbb{R} \backslash \mathbb{Z}, j=1, \ldots, d$. (Note that the case where some of the $c_{j}$ vanish reduces to the direct product of a rotation and a skew product extension over a lower dimensional torus.)

Let $0<\varepsilon(N) \rightarrow 0$. We will say that $\alpha$ admits relatively prime approximation with speed $\varepsilon(N)$ if there exists a sequence of rational vectors $\left(p_{1, n} / N_{n}, \ldots, p_{d, n} / N_{n}\right)$ such that $N_{n} \rightarrow \infty, p_{j, n}$ and $N_{n}$ are relatively prime for every $j=1, \ldots, d$, and

$$
\left|\alpha_{j}-p_{j, n} / N_{n}\right| \leq \varepsilon\left(N_{n}\right)
$$

for $j=1, \ldots, d$.

To see that such approximations exist we prove a lemma which tells us a little more.

Lemma 2. For every sequence of positive numbers $\varepsilon(N) \rightarrow 0$ and every sequence of positive integers $N_{n} \rightarrow \infty$ there exists a residual set $A$ of vectors 
$\alpha$ in $\mathbb{R}^{d}$ such that every $\alpha$ from $A$ admits relatively prime approximation with speed $\varepsilon(N)$ along a subsequence of $N_{n}$.

Pr o of. Define a union of open rectangles

$$
\begin{aligned}
U_{n}= & \bigcup_{p_{1}} \ldots \bigcup_{p_{d}}\left(\frac{p_{1}}{N_{n}}-\varepsilon\left(N_{n}\right), \frac{p_{1}}{N_{n}}+\varepsilon\left(N_{n}\right)\right) \times \ldots \\
& \times\left(\frac{p_{d}}{N_{n}}-\varepsilon\left(N_{n}\right), \frac{p_{d}}{N_{n}}+\varepsilon\left(N_{n}\right)\right),
\end{aligned}
$$

where the $p_{j}$ 's run over all the positive integers less than and relatively prime to $N_{n}$. Now

$$
A=\bigcap_{k=1}^{\infty} \bigcup_{n=k}^{\infty} U_{n}
$$

is a $G_{\delta}$ set and every $\alpha \in A$ has the required property. By Baire's theorem it remains to show that the set $\bigcup_{n=k}^{\infty} U_{n}$ is dense. To see this it clearly suffices to observe that $D(N) / N \rightarrow 0$, where $D(N)$ denotes the maximal distance between two consecutive numbers which are less than and relatively prime to $N$. This, however, can be deduced from the Prime Number Theorem: for any $0<s<t<1$ there are approximately $(t-s) N / \log N$ primes in the interval $[s N, t N)$, while there are only no more than $\log _{2} N$ divisors of $N$ among them. This implies $D(N) \leq(t-s) N$ for all sufficiently large $N$, so $D(N) / N \rightarrow 0$. We note in passing that the best estimate for the maximal distance between two consecutive primes which are less than and relatively prime to $N$ is $O\left(\log ^{2} N\right)$ and was found by H. Iwaniec [5].

Now consider a vector $\alpha=\left(\alpha_{1}, \ldots, \alpha_{d}\right)$ which admits a relatively prime approximation with speed $\varepsilon(N)=o\left(1 / N^{2}\right)$ and with denominators $N_{n}$. We can repeat the calculation of the Fourier coefficients for linear functions made in [7]. Setting $\alpha_{j}-p_{j, n} / N_{n}=\theta_{j, n} / N_{n}^{2}$ we have $\theta_{j, n} \rightarrow 0$ and we obtain, for the linear cocycle $l(x)$,

$$
\widehat{\sigma}\left(N_{n}\right)=C_{n} \prod_{j=1}^{d} \frac{\sin \pi c_{j} \theta_{j, n}}{\pi c_{j} \theta_{j, n}},
$$

where $\left|C_{n}\right| \rightarrow C>0$.

It should be noted that the set of such well-approximable vectors has Lebesgue measure zero in $\mathbb{R}^{d}$; in fact, those which admit a rational approximation with speed $o\left(1 / N^{t}\right)$ for some $t>1+1 / d$ form a null set (see [8]).

On the other hand, since ergodic rotations form a residual set, by Corollary 2 and Lemma 2 we obtain the following. 
THEOREM 2. There exists a residual set $A$ of ergodic rotations $\alpha$ such that for every $\alpha \in A$ and every linear cocycle $l(x)$ with all coefficients noninteger the operator $V$ has continuous singular non-Dirichlet spectrum.

If the linear cocycle has at least one nonzero integer coefficient then for every ergodic rotation $\alpha$ the operator $V$ has Lebesgue spectrum.

If each linear term $c_{j} x_{j}$ in $l(x)$ is perturbed by a 1-periodic absolutely continuous function $g_{j}\left(x_{j}\right)$, the first part of the theorem remains valid, because the sequence $g_{j}^{\left(N_{n}\right)}$ converges uniformly to the constant value $\int g_{j}$ if all the $p_{j, n} / N_{n}$ are in a reduced form and approximate $\alpha_{j}$ with speed $o\left(1 / N^{2}\right)$ (see [4], p. 189).

It would be interesting to decide whether $V$ has singular spectrum for any linear cocycle with noninteger coefficients and every ergodic rotation $\alpha$ of $\mathbb{T}^{d}$ for $d>1$.

3. Ergodicity of cylinder flows. In this section we consider the onedimensional case $d=1$.

Let $\alpha$ be an irrational number. For every measurable function $f: \mathbb{T} \rightarrow \mathbb{R}$ we define the skew product (cylinder flow) $T_{f}$ on $\mathbb{T} \times \mathbb{R}$ by the formula

$$
T_{f}(x, y)=(x+\alpha, y+f(x)) .
$$

Assume that $f$ is piecewise absolutely continuous with $\int f=0$ and $\int f^{\prime} \neq 0$.

In [10] Pask proved that if in addition $f^{\prime}$ is Riemann integrable then $T_{f}$ must be ergodic. Our method presented in the proof of Theorem 1 allows us to show that the same holds true without the additional R-integrability assumption on $f^{\prime}$.

In fact, an analysis of Pask's argument reveals that the R-integrability of $f^{\prime}$ is only used to show that for $n$ sufficiently large the points $x$ satisfying $f^{\left(q_{n}\right)}(x) \in K(\delta)$ take up at least a fixed proportion $W$ of the length of every continuity interval $Q$ of the function $f^{\left(q_{n}\right)}$. Here the compact set $K(\delta)$ equals $[-C, C] \backslash(\eta \mathbb{Z}+(-\delta, \delta))$, where $C$ is a positive constant depending on the total variation of $f$ and $\delta$ is a fixed sufficiently small positive number (see [10], p. 72; we have slighty adapted Pask's notation to ours). Clearly, it suffices to prove the same for each of our "long good intervals" $I$ because they are contained in continuity intervals and, by an appropriate choice of $L$, cover an arbitrarily large part of the unit interval. Now we let $Z=\eta \mathbb{Z}$. Since by the Koksma inequality $f^{\left(q_{n}\right)}$ takes all its values in $[-C, C]$, it follows from the estimate in the proof of our Theorem 1 that the proportion $W$ is no less than $1-(4 / c)(\varepsilon+L M \delta)$. Therefore $W$ can be made as close to 1 as we wish-for all sufficiently small $\delta$.

Consequently, we obtain the following result (cf. [10], 2.11). 
Theorem 3. Let $f: \mathbb{T} \rightarrow \mathbb{R}$ be piecewise absolutely continuous with $\int f=0$ and $\int f^{\prime} \neq 0$. Then the skew product $T_{f}$ is ergodic.

\section{References}

[1] H. Anzai, Ergodic skew product transformations on the torus, Osaka J. Math. 3 (1951), 88-99.

[2] G. H. Choe, Products of operators with singular continuous spectra, in: Proc. Sympos. Pure Math. 51, Amer. Math. Soc., Providence, R.I., 1990, 65-68.

[3] H. Helson, Cocycles on the circle, J. Operator Theory 16 (1986), 189-199.

[4] M. Herman, Sur la conjugaison différentiable des difféomorphismes du cercle à des rotations, Inst. Hautes Etudes Sci. Publ. Math. 49 (1979), 5-234.

[5] H. Iwaniec, On a problem of Jacobsthal, Demonstratio Math. 11 (1978), 225-231.

[6] A. Iwanik, Anzai skew products with Lebesgue component of infinite multiplicity, Bull. London Math. Soc. 29 (1997), 195-199.

[7] A. Iwanik, M. Lemańczyk and C. Mauduit, Piecewise absolutely continuous cocycles over irrational rotations, J. London Math. Soc., to appear.

[8] A. Khintchine, Zur metrischen Theorie der diophantischen Approximationen, Math. Z. 24 (1926), 706-714.

[9] H. A. Medina, Spectral types of unitary operators arising from irrational rotations on the circle group, Michigan Math. J. 41 (1994), 39-49.

[10] D. A. Pask, Skew products over the irrational rotation, Israel J. Math. 69 (1990), 65-74.

Institute of Mathematics

Wrocław University of Technology

Wybrzeże Wyspiańskiego 27

50-370 Wrocław, Poland

E-mail: iwanik@im.pwr.wroc.pl

Received 30 September 1997;

in revised form 22 December 1997 